\title{
HILBERT TRANSFORMS ASSOCIATED WITH PLANE CURVES $\left({ }^{1}\right)$
}

\author{
BY
}

\section{ALEXANDER NAGEL AND STEPHEN WAINGER}

ABSTRACT. Let $(t, \gamma(t))$ be a plane curve. Set $H_{\gamma} f(x, y)=$ p.v. $\int f(x-t, y-\gamma(t)) d t / t$ for $f \in C_{0}^{\infty}\left(R^{2}\right)$. For a large class of curves, the authors prove $\left\|H_{\gamma} f\right\|_{p}<A_{p}\|f\|_{p}, 5 / 3<p<5 / 2$. Various examples are given to show that some condition on the curve $(t, \gamma(t))$ is necessary.

1. Introduction. In [NRW] it was shown that if $\sigma(t)$ is the curve in $\mathbf{R}^{n}$ given by

$$
\sigma(t)=\left(|t|^{\alpha} \operatorname{sgn}(t), \ldots,|t|^{\alpha} n \operatorname{sgn}(t)\right),
$$

with $\alpha_{i}>0$, then the "Hilbert transform along $\sigma$ " given by

$$
T f(x)=\text { p.v. } \int_{-\infty}^{\infty} f(x-\sigma(t)) \frac{d t}{t}
$$

is a bounded linear transformation on $L^{p}\left(\mathrm{R}^{n}\right)$ for $1<p<\infty$. In the same paper it was shown that if $\gamma(t)$ is an odd continuous function of $t$, convex and increasing for $t>0$, equal to $t^{\alpha}$ for $0<t<1$, and equal to at $+b$ for $t$ large, with $b \neq 0$, then the Hilbert transform along the curve $t \rightarrow(t, \gamma(t))$

$$
T f(x, y)=\text { p.v. } \int_{-\infty}^{\infty} f(x-t, y-\gamma(t)) \frac{d t}{t}
$$

is unbounded, even on $L^{2}\left(R^{2}\right)$.

Thus it is clear that the convexity of a function $\gamma(t)$ is not sufficient to insure the boundedness of the operator $T$, defined by (1), on some $L^{p}\left(R^{2}\right)$. The main purpose of the present paper is to study operators of the form (1), and to find sufficient conditions for the boundedness of the operator $T$.

We begin by giving conditions which guarantee that $T$ is bounded on $L^{2}\left(R^{2}\right)$. Our first positive result is

THEOREM 2.1. Suppose $\gamma(t)$ is a continuous odd function of $t$, twice continuously differentiable and convex for $t>0$. Suppose in addition that there exists $\alpha>0$ so that $t^{-\alpha} \gamma^{\prime}(t)$ and $\left(t \gamma^{\prime}(t)\right)^{\prime}$ are monotone increasing. Then $T$ is bounded on $L^{2}\left(\mathbf{R}^{2}\right)$.

Received by the editors June 10, 1975.

AMS (MOS) subject classifications (1970). Primary 42-XX, 44-XX, 44A25.

(1) This research was supported in part by NSF grant \#MPS 75-06687 at the University of Wisconsin, Madison. 
Theorem 2.1 covers a large class of functions $\gamma(t)$ including

$$
\gamma(t)=|t|^{\alpha} \operatorname{sgn} t, \quad \gamma(t)=\operatorname{sgn}(t)\left[e^{|t|}-1\right], \quad \text { or } \gamma(t)=t e^{-1 /|t|} \text {. }
$$

However the theorem does not apply to $\gamma(t)=t \log |t|$, and to deal with such curves, we have

THEOREM 2.2. Suppose $\gamma(t)$ is a continuous odd function of $t$, twice continuously differentiable and convex for $t>0$. Suppose in addition there are constants $C>0$ and $\alpha$ so that (i) $t \gamma^{\prime}(t)-\gamma(t) \leqslant C t^{2} \gamma^{\prime \prime}(t)$, (ii) $t^{\alpha} \gamma^{\prime \prime}(t)$ is monotone, and (iii) $\left(t \gamma^{\prime}(t)\right)^{\prime}$ is monotone increasing. Then $T$ is bounded on $L^{2}\left(R^{2}\right)$.

Both Theorems 2.1 and 2.2 are proved by showing that the associated multiplier $m(x, y)$, defined by $(T f)^{\wedge}(x, y)=m(x, y) \hat{f}(x, y)$, is uniformly bounded on $\mathbf{R}^{2}$. (Here, and throughout this paper, ${ }^{\wedge}$ denotes the Fourier transform.) These estimates are proved in $\$ 2$.

In $\S 3$, we give sufficient conditions on the function $\gamma(t)$ for the operator $T$ to be bounded on $L^{p}\left(R^{2}\right)$ for some range of $p$ about 2 . Our main result is

THEOREM 3.1. Suppose that $\gamma(t)$ is a continuous odd function, twice continuously differentiable, increasing and convex for $t>0$. Suppose in addition that $\gamma^{\prime \prime}(t)$ is monotone for $t>0$ and that there exists $C>0$ so that $\gamma^{\prime}(t) \leqslant$ $C t \gamma^{\prime \prime}(t)$ for $t>0$. Then the operator $T$ is bounded on $L^{p}\left(\mathbf{R}^{2}\right)$ for $5 / 3<p<$ $5 / 2$.

The proof of Theorem 3.1 uses Stein's interpolation theorem, and is similar to the proof of Theorem 1 in [NRW]. We show that certain "roughened" operators remain bounded in $L^{2}\left(\mathbf{R}^{2}\right)$, while sufficiently "smoothed" operators are bounded in $L^{p}\left(\mathbf{R}^{2}\right)$ for $1<p<\infty$. In dealing with these improved operators, we do not have kernels with appropriate homogeneity as in ' $[N R W]$, so we must use the Marcinkiewicz multiplier theorem rather than a Calderon-Zygmund type argument. In effect, this means that our kernels must be smoothed a definite amount, rather than just $\epsilon>0$, and this is why we obtain boundedness in $L^{p}\left(\mathbf{R}^{2}\right)$ only for a proper subinterval of $1<p<\infty$.

It is clear that the hypotheses of Theorem 3.1 are stronger than those of Theorem 2.2. In particular the function $\gamma(t)=t \log |t|$ satisfies the hypotheses of Theorem 2.2 but not those of Theorem 3.1. We show in fact that the method of proof of Theorem 3.1 definitely fails for $t \log |t|$ (Theorem 3.2). In some sense, the difficulty is that $t \log |t|$ is very close to a straight line, and the estimate $\gamma^{\prime}(t) \leqslant C t \gamma^{\prime \prime}(t)$ no longer holds. However, in Theorem 3.3 we show that certain roughened operators do remain bounded in $L^{2}\left(R^{2}\right)$ when $\gamma(t)$ is very close to a straight line. 
Given $0<a<b<\infty$, we can always decompose the operator $T$ defined in (1) by writing:

$$
\begin{aligned}
T f(x, y)= & \text { p.v. } \int_{-a}^{a} f(x-t, y-\gamma(t)) \frac{d t}{t}+\int_{a<|t|<b} f(x-t, y-\gamma(t)) \frac{d t}{t} \\
& + \text { p.v. } \int_{|t|>b} f(x-t, y-\gamma(t)) \frac{d t}{t} \\
= & T_{1} f(x, y)+T_{2} f(x, y)+T_{3} f(x, y) .
\end{aligned}
$$

The operator $T_{2}$ is convolution with a finite measure, and hence is bounded on $L^{p}\left(\mathbf{R}^{2}\right)$ for $1 \leqslant p \leqslant \infty$. In particular, this shows that only the behavior of $\gamma(t)$ near zero and near infinity can affect the boundedness of the operator $T$. While we have stated Theorems $2.1,2.2$, and 3.1 so that they apply only to the full operator $T$, an examination of the proofs will show that they apply as well to the truncated operators $T_{1}$ or $T_{3}$. Thus in checking that a function $\gamma(t)$ satisfies the hypotheses of one of our theorems, it is only necessary to check that the appropriate estimates hold near zero and near infinity.

In particular, it follows from Theorem 3.1 that if $\gamma(t)$ is real analytic, the truncated operator $T_{1}$ is bounded on $L^{p}\left(\mathbf{R}^{2}\right)$ for $5 / 3<p<5 / 2$. Thus it is reasonable to ask if sufficient smoothness of the function $\gamma(t)$ near 0 implies the boundedness of the truncated operator $T_{1}$. However in $\$ 4$ we show

ThEOREM 4.1. There is an odd, $C^{\infty}$-function $\gamma(t)$, defined for $-1 \leqslant t \leqslant$ +1 , increasing and convex for $t>0$ such that

$$
T_{1} f(x, y)=\text { p.v. } \int_{-1}^{+1} f(x-t, y-\gamma(t)) \frac{d t}{t}
$$

is unbounded on $L^{2}\left(R^{2}\right)$.

Again, it might be asked, especially in view of the counterexample in [NRW], if sufficiently rapid growth of $\gamma^{\prime \prime}(t)$ as $t$ gets large insures the boundedness of the operator $T_{3}$. However, we show

THEOREM 4.2. Let $\psi(t)$ be any increasing function of $t$. Then there exists an odd, $C^{\infty}$-function $\gamma(t)$, increasing and convex for $t>0$, with $\gamma^{\prime \prime}(t) \geqslant \psi(t)$ such that $T_{3} f(x, y)=$ p.v. $\int_{|t| \geqslant 1} f(x-t, y-\gamma(t)) d t / t$ is unbounded on $L^{2}\left(\mathrm{R}^{2}\right)$.

The examples for Theorems 4.1 and 4.2 are constructed by using "partition of unity" type arguments. However, it is not necessary to leave the class of elementary functions to find cases where $T_{3}$ is unbounded. We show, for example, that

$$
S f(x, y)=\operatorname{p.v} \cdot \int_{|t| \geqslant 2} f(x-t, y-\operatorname{sgn}(t) \log |t|) \frac{d t}{t}
$$

is unbounded in $L^{2}\left(\mathbf{R}^{2}\right)$. (In contrast, note that the arguments of Theorem 3.1 show that 


$$
R f(x, y)=\text { p.v. } \int_{|t| \geqslant 2} f\left(x, t, y-\operatorname{sgn}(t) e^{|t|}\right) \frac{d t}{t}
$$

is bounded on $L^{p}\left(\mathbf{R}^{2}\right)$ for $5 / 3<p<5 / 2$.) We also show that the associated maximal function

$$
M f(x, y)=\sup _{h>1} \frac{1}{2 h} \int_{-h}^{+h}|f(x-t, y-\log |t|)| d t
$$

does not define a bounded operator on any $L^{p}\left(\mathbf{R}^{2}\right), p<\infty$.

2. Boundedness in $L^{2}\left(R^{2}\right)$. In this section, we obtain sufficient conditions for the operator $T$ defined in (1) to be bounded on $L^{2}\left(R^{2}\right)$. Throughout this section, $\gamma(t)$ will denote a continuous odd function of $t$, which is convex, and twice continuously differentiable for $t>0$. Our main results are:

THEOREM 2.1. Suppose there exists $\alpha>0$ so that $t^{-\alpha} \gamma^{\prime}(t)$ and $\left(t \gamma^{\prime}(t)\right)^{\prime}$ are monotone increasing. Then $T$ is bounded on $L^{2}\left(\mathbf{R}^{2}\right)$.

THEOREM 2.2. Suppose that there are constants $C>0$ and $\alpha$ so that

(i) $t \gamma^{\prime}(t)-\gamma(t) \leqslant C t^{2} \gamma^{\prime \prime}(t)$,

(ii) $t^{\alpha} \gamma^{\prime \prime}(t)$ is monotone increasing,

(iii) $\left(t \gamma^{\prime}(t)\right)^{\prime}$ is monotone increasing.

Then the operator $T$ is bounded on $L^{2}\left(R^{2}\right)$.

Both of these results will be obtained as corollaries of the more general result:

THEOREM 2.3. Suppose there exists $C>0$ so that for all $\xi>0$ and all $t>0$ with $t \notin[1 / 2 \xi, 2 \xi]$,

$$
t \gamma^{\prime}(t)-\gamma(t) \leqslant C t\left|\gamma^{\prime}(t)-\gamma^{\prime}(\xi)\right| .
$$

Suppose also that $\left(\operatorname{t}^{\prime}(t)\right)^{\prime}$ is monotone increasing. Then $T$ is bounded on $L^{2}\left(\mathbf{R}^{2}\right)$.

Clearly, the boundedness of the operator $T$ on $L^{2}\left(R^{2}\right)$ is equivalent to the uniform boundedness of the corresponding multiplier $m(x, y)$, where $(T f)^{\wedge}(x, y)$ $=m(x, y) \hat{f}(x, y)$. An easy calculation shows that

$$
m(x, y)=\text { p.v. } \int_{0}^{\infty} \sin (x t+y \gamma(t)) \frac{d t}{t} .
$$

We shall need the following elementary result:

LeMma 2.1. Let $A<B$, let $h \in L^{\infty}((A, B)) \cap C^{1}((A, B))$ and suppose $(h(t) / t)^{\prime}=0$ has at most $k$ roots in $(A, B)-(-1,1)$. Then

$$
\left|\int_{A}^{B} \sin (t) h(t) \frac{d t}{t}\right| \leqslant(2+4 k)\|h\|_{\infty} .
$$


Proof. We certainly have $\left|S_{-1}^{+1} \sin (t) h(t) d t / t\right| \leqslant 2\|h\|_{\infty}$. Next, if $t_{j}, t_{j+1}$ are successive roots of $(h(t) / t)^{\prime}=0$, so that $h(t) / t$ is monotone on $\left(t_{j}, t_{j+1}\right)$, the second mean value theorem gives

$$
\left|\int_{t_{j}}^{t_{j+1}} \sin (t) h(t) \frac{d t}{t}\right|=\left|\int_{t_{j}}^{t_{j+1}} \frac{h(t)}{t} d(-\cos (t))\right| \leqslant 2\|h\|_{\infty}\left(\frac{1}{\left|t_{j}\right|}+\frac{1}{\left|t_{j+1}\right|}\right) .
$$

The lemma follows by adding these estimates.

We turn now to the proof of Theorem 2.3. Suppose that $x, y \geqslant 0$, and consider

$$
m(x,-y)=\int_{0}^{\infty} \sin (x t-y \gamma(t)) \frac{d t}{t}=\int_{0}^{1 / 2 \xi}+\int_{1 / 2 \xi}^{2 \xi}+\int_{2 \xi}^{\infty}
$$

where $\xi$ satisfies $\gamma^{\prime}(\xi)=x / y$. Note that $\left|\int_{1 / 2 \xi}^{2 \xi} \sin (x t-y \gamma(t)) d t / t\right| \leqslant \log (4)$, while $(x t-y \gamma(t))$ is monotone increasing on the interval $(0,1 / 2 \xi)$, and monotone decreasing on the interval $(2 \xi, \infty)$. Hence the function $s(t)=x t-y \gamma(t)$ has an inverse on each of these intervals, and we write this inverse as $t=\mu(s)$ in both cases. We then have:

$$
\begin{aligned}
& \int_{0}^{1 / 2 \xi} \sin (x t-y \gamma(t)) \frac{d t}{t}=\int_{0}^{s(1 / \xi \xi)} \sin (s)\left[\frac{s \mu^{\prime}(s)}{\mu(s)}\right] \frac{d s}{s}, \\
& \int_{2 \xi}^{\infty} \sin (x t-y \gamma(t)) \frac{d t}{t}=\int_{s(2 \xi)}^{-\infty} \sin (s)\left[\frac{s \mu^{\prime}(s)}{\mu(s)}\right] \frac{d s}{s} .
\end{aligned}
$$

Note that on $(0, s(1 / 2 \xi))$ and on $\left.(-\infty, s(2 \xi)),\left(\mu^{\prime}(s) / \mu(s)\right)^{\prime}=(\log \mu(s))\right)^{\prime \prime}=0$ has a root if and only if $\left(x e^{t}-y \gamma\left(e^{t}\right)\right)^{\prime \prime}=0$ has a root, and this last equation has at most one root for any value of $x$ and $y$ by the hypothesis on $\left(t \gamma^{\prime}(t)\right)^{\prime}$. It thus follows by Lemma 2.1 that to show that $m(x, y)$ is bounded, it suffices to show that $\left(s \mu^{\prime}(s) / \mu(s)\right)$ is uniformly bounded for $s \notin[s(1 / 2 \xi), s(2 \xi)]$. This is the same as showing that $(x t-y \gamma(t)) / t\left(x-y \gamma^{\prime}(t)\right)$ is uniformly bounded (independent of $x$ and $y)$ for $t \notin\left[12 \frac{2}{2}, 2 \xi\right]$. But

$$
\begin{aligned}
\frac{x t-y \gamma(t)}{t\left(x-y^{\prime} \gamma(t)\right)} & =\frac{(x / y) t-\gamma(t)}{t\left[(x / y)-\gamma^{\prime}(t)\right]}=\frac{\gamma^{\prime}(\xi) t-\gamma(t)}{t \gamma^{\prime}(\xi)-t \gamma^{\prime}(t)} \\
& =1+\frac{t \gamma^{\prime}(t)-\gamma(t)}{t\left[\gamma^{\prime}(\xi)-\gamma^{\prime}(t)\right]} .
\end{aligned}
$$

The hypothesis of Theorem 2.3 thus shows that $m(x, y)$ is uniformly bounded if $x \geqslant 0, y \leqslant 0$. The quadrant where $x \leqslant 0, y \geqslant 0$ is handled similarly, and the two quadrants where $x$ and $y$ have the same sign are even easier, since then $x t+$ $y \gamma(t)$ is then monotone on $(0, \infty)$. Thus Theorem 2.3 is proved.

We turn next to the proofs of Theorems 2.1 and 2.2. To prove Theorem 2.1 , suppose $t^{-\alpha} \gamma^{\prime}(t)$ is monotone increasing. If $t \leqslant 1 / 2 \xi$, we have $t^{-\alpha} \gamma^{\prime}(t) \leqslant$ 
$\xi^{-\alpha} \gamma^{\prime}(\xi)$ and hence $\gamma^{\prime}(\xi) \geqslant(\xi / t)^{\alpha} \gamma^{\prime}(t) \geqslant 2^{\alpha} \gamma^{\prime}(t)$. But then

$$
\frac{t \gamma^{\prime}(t)-\gamma(t)}{t\left[\gamma^{\prime}(\xi)-\gamma(t)\right]} \leqslant \frac{t \gamma^{\prime}(t)-\gamma(t)}{t \gamma^{\prime}(t)\left[2^{\alpha}-1\right]}<\frac{1}{2^{\alpha}-1}
$$

A similar argument works if $t \geqslant 2 \xi$, and thus $t^{-\alpha} \gamma^{\prime}(t)$ monotone increasing implies condition (*). Thus Theorem 2.1 is proved.

To prove Theorem 2.2, suppose that

(i) $t \gamma^{\prime}(t)-\gamma(t) \leqslant C t^{2} \gamma^{\prime \prime}(t)$, and

(ii) $t^{\alpha} \gamma^{\prime \prime}(t)$ is monotone increasing.

Note that

$$
\frac{t \gamma^{\prime}(t)-\gamma(t)}{t \gamma^{\prime}(t)-t \gamma^{\prime}(\xi)}=1-\frac{\xi \gamma^{\prime}(\xi)-\gamma(\xi)}{\xi \gamma^{\prime}(\xi)-\xi \gamma^{\prime}(t)}-\frac{\gamma(t) / t-\gamma(\xi) / \xi}{\gamma^{\prime}(t)-\gamma^{\prime}(\xi)}
$$

By the generalized mean value theorem, the last of these three terms is, in absolute value, equal to $\left|\left(\eta \gamma^{\prime}(\eta)-\gamma(\eta)\right) / \eta^{2} \gamma^{\prime \prime}(\eta)\right|$ where $\eta$ is between $t$ and $\xi$, and this is uniformly bounded by (i). But the second term in (2) is the same as the left-hand side of (2), with the roles of $\xi$ and $t$ interchanged. Hence it suffices to show that $\left(t \gamma^{\prime}(t)-\gamma(t)\right) /\left(t \gamma^{\prime}(t)-t \gamma^{\prime}(a)\right)$ is bounded when $t<1 / 2 a$.

Now assume that $t^{\alpha} \gamma^{\prime \prime}(t)$ is monotone increasing. Without loss of generality, $\alpha>1$, we have

$$
\begin{aligned}
\gamma^{\prime}(a)-\gamma^{\prime}(t) & =\int_{t}^{a} \gamma^{\prime \prime}(s) d s \geqslant t^{\alpha} \gamma^{\prime \prime}(t) \int_{t}^{a} s^{-\alpha} d s \\
& =\frac{1}{(\alpha-1)} t \gamma^{\prime \prime}(t)\left[1-\left(\frac{a}{t}\right)^{1-\alpha}\right] \geqslant\left(\frac{1-2^{1-\alpha}}{\alpha-1}\right) t \gamma^{\prime \prime}(t) .
\end{aligned}
$$

Then

$$
\left|\frac{t \gamma^{\prime}(t)-\gamma(t)}{t \gamma^{\prime}(t)-t \gamma^{\prime}(\xi)}\right| \leqslant\left(\frac{\alpha-1}{1-2^{1-\alpha}}\right) \frac{t \gamma^{\prime}(t)-\gamma(t)}{t^{2} \gamma^{\prime \prime}(t)} \leqslant C
$$

by hypothesis (i), and this completes the proof of Theorem 2.2 .

3. Boundedness in $L^{p}\left(\mathbf{R}^{2}\right)$. In this section, we show that there is a large class of curves $\gamma(t)$ such that the operator $T$ defined in (1) is bounded on $L^{p}\left(\mathbf{R}^{2}\right)$ for a range of $p$ about 2 . Throughout this section we shall make the following hypotheses about the function $\gamma(t)$ :

(i) $\gamma(t)$ is a continuous, odd function, convex and increasing for $t>0$.

(ii) $\gamma$ is twice continuously differentiable for $t>0, \gamma^{\prime \prime}(t)>$ 0 , and $\gamma^{\prime \prime}(t)$ is monotone for $t>0$.

(iii) There exists $C>0$ so that $\gamma^{\prime}(t) \leqslant C t \gamma^{\prime \prime}(t)$ for $t>0$.

We note that condition (iii) follows from condition (ii) if $\gamma^{\prime \prime}(t)$ is monotone increasing, but does not follow in general if $\gamma^{\prime \prime}(t)$ is decreasing (as for example in the case $\gamma(t)=t \log |t|)$. We also note that since $\gamma^{\prime}(t)$ is monotone increasing, 
we obtain the additional estimates:

(iv)

$$
\gamma(t) \leqslant C t \gamma^{\prime}(t) \text { and } \gamma(t) \leqslant C t^{2} \gamma^{\prime \prime}(t) \text { for some } C>0 \text {. }
$$

The main result of this section is

THEOREM 3.1. Under the hypotheses (i), (ii), and (iii), the operator $T$, defined in (1), is bounded on $L^{p}\left(\mathbf{R}^{2}\right)$ for $5 / 3<p<5 / 2$.

In proving Theorem 3.1, we shall need the following lemmas:

LEMMA 3.1 (VAN DER CORPUT). Let $f(t)$ be a twice continuously differentiable function on $a \leqslant t \leqslant b$.

(i) If $f^{\prime}(t)$ is monotone, and $\left|f^{\prime}(t)\right| \geqslant \lambda$ for $a \leqslant t \leqslant b$, then

$$
\left|\int_{a}^{b} \operatorname{expif}(t) d t\right| \leqslant C \lambda^{-1}
$$

(ii) If $\left|f^{\prime \prime}(t)\right| \geqslant \mu$ for $a \leqslant t \leqslant b$, then

$$
\left|\int_{a}^{b} \exp i f(t) d t\right| \leqslant C \mu^{-1 / 2} \text {. }
$$

Here $C$ is a constant independent of $a, b$, and $f$. A proof of this lemma can be found in [Z, Vol. 1, p. 197].

LEMMA 3.2. Let $g(t)$ be a function of $t$ satisfying

(a) $|g(t)| \leqslant A_{1}|t|$ for $|t| \leqslant 1$.

(b) $|g(t)| \leqslant A_{2}|t|^{-\eta}$ for $|t| \geqslant 1$ and some $\eta>0$.

Then there exists $C>0$, independent of $x, y, a, b$, so that

$$
\left|\int_{a}^{b} \exp i[x t+y \gamma(t)] g(y \gamma(t)) \frac{d t}{t}\right| \leqslant C\left[A_{1}+A_{2}\right] .
$$

Proof. For $y$ fixed, choose $t_{0}>0$ (depending on $y$ ) so that $|y| \gamma\left(t_{0}\right)=$ 1. Now

$$
\begin{aligned}
\left|\int_{a}^{b} \exp i[x t+y \gamma(t)] g(y \gamma(t)) \frac{d t}{t}\right| & \leqslant \int_{a}^{b}|g(y \gamma(t))| \frac{d t}{|t|} \\
& =\int_{|t|<t_{0} ; t \in[a, b]}+\int_{|t|>t_{0} ; t \in[a, b]} .
\end{aligned}
$$

Now using (a),

$$
\begin{aligned}
\int_{0}^{t_{0}}|g(y \gamma(t))| \frac{d t}{t} & \leqslant A_{1}|y| \int_{0}^{t_{0}} \gamma(t) \frac{d t}{t} \\
& \leqslant A_{1} C|y| \int_{0}^{t_{0}} \gamma^{\prime}(t) d t \quad \text { by (iv) } \\
& =A_{1} C .
\end{aligned}
$$


Using (b),

$$
\begin{aligned}
\int_{t_{0}}^{b}|g(y \gamma(t))| \frac{d t}{t} & \leqslant A_{2}|y|^{-\eta} \int_{t_{0}}^{b} \gamma(t)^{-\eta} \frac{d t}{t} \\
& \leqslant A_{2} C|y|^{-\eta} \int_{t_{0}}^{b} \gamma(t)^{-\eta-1} \gamma^{\prime}(t) d t \quad \text { by (iv) } \\
& \leqslant A_{2} C|y|^{-\eta} \int_{1 / y}^{\gamma(b)} u^{-\eta-1} d u \leqslant A_{2} C .
\end{aligned}
$$

This completes the proof.

LEммA 3.3. Let $g(t)$ be an even, differentiable function of $t$ satisfying

(a) $|g(t)| \leqslant A_{1}$, for $|t| \leqslant 1$,

(b) $g^{\prime}(t)=0$ has only a finite number of roots for $|t| \leqslant 1$,

(c) $|g(t)| \leqslant A_{2}|t|^{\eta}$ for $|t| \geqslant 1$ and some $\eta \in(-\infty,+1 / 2)$,

(d) $\left|g^{\prime}(t)\right| \leqslant A_{3}|t|^{\eta-1}$ for $|t| \geqslant 1$.

Then there exists $C>0$, independent of $x, y$, and $a$, so that:

$$
\mid \text { p.v. } \int_{-a}^{+a} \exp i[x t+y \gamma(t)] g(y \gamma(t)) \frac{d t}{t} \mid \leqslant C\left[A_{1}+A_{2}+A_{3}\right] .
$$

Proof. Again for $y$ fixed, choose $t_{0}>0$ so that $|y| \gamma\left(t_{0}\right)=1$. For any $\epsilon<t_{0}$, we have:

$$
\begin{aligned}
\left|\int_{\epsilon<|t|<t_{0}} \exp i[x t+y \gamma(t)] g(y \gamma(t)) \frac{d t}{t}\right| \leqslant & \left|\int_{\epsilon<|t|<t_{0}} \exp i[x t] g(y \gamma(t)) \frac{d t}{t}\right| \\
& +\int_{\epsilon<|t|<t_{0}}|y||\gamma(t)| \lg (y \gamma(t)) \mid \frac{d t}{|t|}
\end{aligned}
$$

The second integral is easily seen to be bounded by $C A_{1}$, by using hypothesis (a), and estimate (iv). On the other hand, for $|t| \leqslant t_{0}$ the function $t \rightarrow g(y \gamma(t))$ is uniformly bounded, and piecewise monotone by hypothesis (b), so by the second mean value theorem, the first integral is bounded by $C A_{1}$.

We next deal with the part of the integral where $|t| \geqslant t_{0}$. If $\gamma^{\prime \prime}(t)$ is monotone increasing, put

$$
\varphi(t)=-\int_{t}^{\infty} \exp i[x s+y \gamma(s)] d s,
$$

while if $\gamma^{\prime \prime}(t)$ is monotone decreasing, put

$$
\varphi(t)=\int_{t_{0}}^{t} \exp i[x s+y \gamma(s)] d s .
$$

Then $d \varphi(t) / d t=\exp i[x t+y \gamma(t)]$, and Lemma 3.1 shows that in either case $|\varphi(t)| \leqslant C\left(|y| \gamma^{\prime \prime}(t)\right)^{-1 / 2}$. Now 


$$
\begin{aligned}
\int_{t_{0}}^{a} \frac{d \varphi}{d t}(t) g(y \gamma(t)) \frac{d t}{t} & =\left.\varphi(t) g(y \gamma(\mathrm{t})) \frac{1}{t}\right|_{t_{0}} ^{a} \\
& -\int_{t_{0}}^{a} \varphi(t)\left[\frac{g^{\prime}(y \gamma(t)) y \gamma^{\prime}(t)}{t}-\frac{g(y \gamma(t))}{t^{2}}\right] d t .
\end{aligned}
$$

For the boundary terms we have

$$
\begin{aligned}
\left|\varphi(t) g(y \gamma(t)) t^{-1}\right| & \leqslant C|y|^{-1 / 2} \gamma^{\prime \prime}(t)^{-1 / 2} A_{2}|y|^{\eta} \gamma(t)^{\eta} \cdot 1 / t \\
& \leqslant C A_{2}|y|^{\eta-1 / 2} \gamma(t)^{\eta-1 / 2} \text { by (iv). }
\end{aligned}
$$

Since $\eta<1 / 2$, and $\gamma(t)$ is increasing, this shows that the contribution from the boundary terms is bounded by $C A_{2}$. On the other hand, the integral term is bounded by

$$
\begin{aligned}
C \int_{t_{0}}^{a}|y|^{-1 / 2} \gamma^{\prime \prime}(t)^{-1 / 2}\left[\frac{A_{3}|y|^{\eta} \gamma(t)^{\eta-1} \gamma^{\prime}(t)}{t}+\frac{A_{2}|y|^{\eta} \gamma(t)^{\eta}}{t^{2}}\right] d t \\
\quad \leqslant C A_{3}|y|^{\eta-1 / 2} \int_{t_{0}}^{a} \gamma(t)^{\eta-3 / 2} \gamma^{\prime}(t) d t+C A_{2}|y|^{\eta-1 / 2} \int_{t_{0}}^{a} \gamma(t)^{\eta-3 / 2} \gamma^{\prime}(t) d t \\
\quad \leqslant C A_{2}+C A_{3} .
\end{aligned}
$$

The integral $\int_{-a}^{-t_{0}}(d \varphi(t) / d t) g(y \gamma(t)) d t / t$ is handled similarly, and this completes the proof of Lemma 3.3.

LEMMA 3.4. Let $g(t)$ be a function of $t$ such that $\int_{-\infty}^{\infty}|g(t)| d t \leqslant C$. Then

ProOF.

$$
\left|\int_{a}^{b} \exp i[x t+y \gamma(t)] y \gamma^{\prime}(t) g(y \gamma(t)) d t\right| \leqslant C \text {. }
$$

$$
\left|\int_{a}^{b} \exp i[x t+y \gamma(t)] y \gamma^{\prime}(t) g(y \gamma(t)) d t\right| \leqslant \int_{a}^{b}|y| \gamma^{\prime}(t)|g(y \gamma(t))| d t .
$$

Now making the change of variables $u=y \gamma(t)$, we obtain the required estimate.

We now turn to the proof of Theorem 3.1. Our argument is similar to that in the proof of Theorem 1 in [NRW]. We introduce an analytic family of operators $T_{z}$ determined by

$$
\left(T_{z} f\right)^{\wedge}(x, y)=m_{z}(x, y) \hat{f}(x, y)
$$

where

$$
m_{z}(x, y)=\text { p.v. } \int_{-\infty}^{\infty} \exp i[x t+y \gamma(t)]\left(1+y^{2} \gamma(t)^{2}\right)^{z} \frac{d t}{t} .
$$

Thus $m_{0}$ is the multiplier corresponding to our original operator $T$. We will show:

(A) If $\epsilon>0$ and if $\operatorname{Re}(z)=1 / 4-\epsilon$, the operator $T_{z}$ is still bounded on $L^{2}\left(\mathbf{R}^{2}\right)$ with operator norm $\left\|T_{z}\right\|_{L^{2}} \leqslant C_{\epsilon}(1+|\operatorname{Im}(z)|)$, where $C_{\epsilon}$ depends only on $\epsilon$. 
(B) If $\operatorname{Re}(z)<-1$, the operator $T_{z}$ is bounded on $L^{p}\left(\mathrm{R}^{2}\right)$ for $1<p<$ $\infty$, with operator norm $\left\|T_{z}\right\|_{L^{p}} \leqslant C(1+|\operatorname{Im}(z)|)$ where $C$ depends only on $\operatorname{Re}(z)$.

The theorem follows from (A) and (B) by using Stein's complex interpolation theorem (see [SW, p. 205]). To prove (A), let $g(t)=\left(1+t^{2}\right)^{2}$. Then $g(t)$ satisfies Lemma 3.3 with $\eta=2 \operatorname{Re}(z), A_{1}$ and $A_{2}$ constants depending only on $\operatorname{Re}(z)$, and $A_{3} \leqslant C(\operatorname{Re}(z)+|\operatorname{Im}(z)|)$. It then follows from Lemma 3.3 that if $\operatorname{Re}(z)=1 / 4-\epsilon, m_{z}(x, y)$ is uniformly bounded on $\mathrm{R}^{2}$, with bound $C(1+|\operatorname{Im}(z)|)$ where $C$ depends only on $\epsilon$. This is equivalent to statement (A).

To prove statement (B), we will show that for $\operatorname{Re}(z)<-1$, the functions

$$
m_{z}(x, y), x \frac{\partial m_{z}}{\partial x}(x, y), y \frac{\partial m_{z}}{\partial y}(x, y) \text {, and } x y \frac{\partial^{2} m_{z}}{\partial x \partial y}(x, y)
$$

are uniformly bounded on $\mathbf{R}^{\mathbf{2}}$. It then follows from the Marcinkiewicz multiplier theorem that $T_{z}$ is bounded on $L^{p}\left(\mathbf{R}^{2}\right), 1<p<\infty$. (See [S, p. 96].)

Using the definition of $m_{z}(x, y)$, we see that we must show that each of the following six integrals is uniformly bounded in $x$ and $y$ if $\operatorname{Re}(z)<-1$ :

$$
\begin{aligned}
& \text { p.v. } \int_{-\infty}^{\infty} \exp i[x t+y \gamma(t)]\left(1+y^{2} \gamma(t)^{2}\right)^{z} \frac{d t}{t}, \\
& \text { p.v. } \int_{-\infty}^{\infty} \exp i[x t+y \gamma(t)] x\left(1+y^{2} \gamma(t)^{2}\right)^{z} d t, \\
& \text { p.v. } \int_{-\infty}^{\infty} \exp i[x t+y \gamma(t)](y \gamma(t))\left(1+y^{2} \gamma(t)^{2}\right)^{z} \frac{d t}{t}, \\
& \text { p.v. } \int_{-\infty}^{\infty} \exp i[x t+y \gamma(t)] z\left(1+y^{2} \gamma(t)^{2}\right)^{z-1} \cdot 2 y^{2} \gamma(t)^{2} \frac{d t}{t}, \\
& \text { p.v. } \int_{-\infty}^{\infty} \exp i[x t+y \gamma(t)] x(y \gamma(t))\left(1+y^{2} \gamma(t)^{2}\right)^{2} d t, \\
& \text { p.v. } \int_{-\infty}^{\infty} \exp i[x t+y \gamma(t)] x z\left(1+y^{2} \gamma(t)^{2}\right)^{2-1} \cdot 2 y^{2} \gamma(t)^{2} d t .
\end{aligned}
$$

The boundedness of (1) follows from Lemma 3.3 with $g(t)=\left(1+t^{2}\right)^{2}$. The boundedness of (3) and (4) follows from Lemma 3.2 with $g(t)=t\left(1+t^{2}\right)^{z}$ or $g(t)=2 z t^{2}\left(1+t^{2}\right)^{z-1}$. To take care of (2), (5), and (6) we note that they all can be written

$$
\text { p.v. } \int_{-\infty}^{\infty} \frac{d}{d t}\left(e^{i x t}\right) e^{i y \gamma(t)} h(y \gamma(t)) d t
$$

with $h(t)$ respectively $\left(1+t^{2}\right)^{z}, t\left(1+t^{2}\right)^{z}$, and $2 z t^{2}\left(1+t^{2}\right)^{z-1}$. Integrating by parts, we obtain

$$
\begin{aligned}
\exp i[x t+ & y \gamma(t)]\left.h(y \gamma(t))\right|_{-\infty} ^{\infty} \\
& - \text { p.v. } \int_{-\infty}^{\infty} \exp i[x t+y \gamma(t)]\left[y \gamma^{\prime}(t) h(y \gamma(t))+y \gamma^{\prime}(t) h^{\prime}(y \gamma(t))\right] d t .
\end{aligned}
$$

As long as $h(t)$ is uniformly bounded, the contribution from the boundary terms 
is bounded. The boundedness of the integrated terms follows from Lemma 3.4, with $g(t)=h(t)$ or $g(t)=h^{\prime}(t)$. Note that $g(t)$ is integrable provided $1+2 \operatorname{Re}(z)$ $<-1$ or $\operatorname{Re}(z)<-1$. This then completes the proof of the theorem.

It is clear from our proof that the same theorem holds for the truncated operators $T_{1}$ or $T_{3}$ defined in the introduction. In particular, we obtain

COROLlARY. Let $\gamma(t)$ be a real analytic function on $[0,1]$, with $\gamma(0)=$ 0. Then

$$
T f(x, y)=\text { p.v. } \int_{-1}^{+1} f(x-t, y-\operatorname{sgn}(t) \gamma(|t|)) \frac{d t}{t}
$$

is bounded on $L^{p}\left(\mathbf{R}^{2}\right)$ for $5 / 3<p<5 / 2$.

Proof. After making a change of variables in $x$ and $y$, we can assume $\gamma^{\prime}(0)=0$. We can write $\gamma(t)=t^{N} \varphi(t)$ where $N \geqslant 2, \varphi(t)$ is real analytic, and $\varphi(0) \neq 0$. Without loss of generality, we can assume $\varphi(0)>0$. Then since $\varphi$ is real analytic, we can find an interval $(0, a)$ on which we have $\varphi^{\prime}(t)>0, \varphi^{\prime \prime}(t)>$ $0, \varphi^{\prime}(t) \leqslant C t \varphi^{\prime \prime}(t)$. The Corollary then follows from Theorem 3.1.

It was pointed out in the introduction that the curve $\gamma(t)=t \log |t|$ satisfies the hypotheses of Theorem 2.2 but not those of Theorem 3.1. We now show that the method of proof of Theorem 3.1 is sharp in the sense that for $\gamma(t)=t \log |t|$, the worsened operators fail to be bounded in $L^{2}\left(\mathbf{R}^{2}\right)$. More precisely, we show

THEOREM 3.2. Let $\delta>0$. Then

$$
m_{\delta}(x, y)=\text { p.v. } \int_{-\infty}^{\infty} \exp i[x t+y t \log |t|]\left(1+y^{2} t^{2} \log ^{2}|t|\right)^{\delta} \frac{d t}{t}
$$

is unbounded on $\mathbf{R}^{2}$.

Proof. If we make the change of variables $t=s e^{-x / y}$, and if we let $r=$ $y e^{-x / y}, R=-x / y$, we obtain

$$
m_{\delta}(x, y)=\int_{0}^{\infty} \sin (r s \log s)\left(1+r^{2} s^{2}(\log (s)+R)^{2}\right)^{\delta} \frac{d s}{s} .
$$

We shall show that for each fixed $r$,

$$
m_{\delta}(x, y)=c(r) R^{2 \delta} r^{2 \delta}+o\left(R^{2 \delta}\right)
$$

as $R$ tends to infinity, where

$$
c(r)=\int_{0}^{\infty} \sin (r \log s) s^{2 \delta-1} d s .
$$

We shall then complete the proof by showing that $c(r)$ tends to infinity as $r$ approaches zero, and hence $c(r)$ is not identically zero.

Write 


$$
\begin{aligned}
m_{\delta}(x, y)= & \int_{0}^{R^{1 / 4}} \sin (r s \log s)\left(1+r^{2} s^{2}(\log (s)+R)^{2}\right)^{\delta} \frac{d s}{s} \\
& +\int_{R^{1 / 4}}^{\infty} \sin (r s \log s)\left(1+r^{2} s^{2}(\log (s)+R)^{2}\right)^{\delta} \frac{d s}{s} \\
= & A(r, R)+B(r, R) .
\end{aligned}
$$

Then

$$
\begin{aligned}
A & =(r R)^{2 \delta} \int_{0}^{R^{1 / 4}} \sin (r s \log s)\left\{\frac{1}{R^{2} r^{2} s^{2}}+\left(\frac{\log (s)}{R}+1\right)^{2}\right\}^{\delta} s^{2 \delta} \frac{d s}{s} \\
& =(r R)^{2 \delta} \int_{0}^{R^{1 / 4}} \sin (r s \log s) s^{2 \delta} \frac{d s}{s}+o\left(R^{2 \delta}\right) \quad \text { as } R \rightarrow \infty
\end{aligned}
$$

By Van der Corput's lemma (Lemma 3.1), we have

$$
\int_{t}^{\infty} \sin (r s \log s) d s \leqslant \frac{C}{r \log t} .
$$

Hence an integration by parts shows that

$$
\int_{R^{1 / 4}}^{\infty} \sin (r s \log s) s^{2 \delta-1} d s=O\left\{\frac{R^{\delta / 2-1 / 4}}{\log R}\right\}
$$

as $R \rightarrow \infty$. Hence

$$
A(r, R)=(r R)^{2 \delta} c(r)+o\left(R^{2 \delta}\right) \quad \text { as } R \rightarrow \infty .
$$

It remains to show that $B(r, R)=o\left(R^{2 \delta}\right)$. This is proved similarly, by using Van der Corput's lemma, and integrating by parts. Hence to complete the proof of the theorem, it only remains to show that $c(r)$ tends to infinity as $r$ tends to zero.

Clearly, as $r$ tends to zero,

$$
\begin{aligned}
c(r)= & O(1)+\int_{1}^{\infty} \sin [r s \log s] s^{2 \delta-1} d s \\
= & O(1)+\frac{1}{r} \int_{1}^{\infty} \frac{s^{2 \delta-1}}{1+\log (s)} \frac{d}{d s}(1-\cos (r s \log s)) d s \\
= & O(1)+\frac{1}{r} \int_{1}^{\infty}(1-\cos (r s \log s)) \\
& \times\left\{(1-2 \delta) s^{2 \delta-2}+\frac{s^{2 \delta-2}}{1+\log (s)}\right\} \frac{d s}{1+\log (s)} .
\end{aligned}
$$

Now for any positive $\epsilon$, this last integral is larger than

$$
\frac{c}{r} \int_{1}^{(1 / r)^{1-\epsilon}} s^{2 \delta-2-\epsilon} d s \geqslant \frac{c}{r^{\eta}} \text { for some } \eta>0 \text {. }
$$

This shows that $c(r)$ tends to infinity, and completes the proof of Theorem 3.2. 
Thus we see that when $\gamma$ is very close to a straight line, and in particular, when the estimate $\gamma^{\prime}(t)<C t \gamma^{\prime \prime}(t)$ fails, the worsened operators in Theorem 3.1 can fail to be bounded in $L^{2}\left(\mathbf{R}^{2}\right)$. It is possible, however, to prove $L^{2}\left(\mathbf{R}^{2}\right)$ boundedness for certain modified worsened operators for curves close to a straight line. For simplicity, we shall only deal with the case of $\operatorname{Im}(z)=0$.

THEOREM 3.3. Let $\gamma(t)$ be a continuous odd function of $t$, twice continuously differentiable and convex for $t>0$. Suppose there exists $C>0$ and $\alpha<2$ so that

(i) $\gamma^{\prime \prime}(t)$ is monotone decreasing for $t>0$,

(ii) $t^{\alpha} \gamma^{\prime \prime}(t)$ is monotone increasing for $t>0$,

(iii) $t \gamma^{\prime}(t)-\gamma(t)<C t^{2} \gamma^{\prime \prime}(t)$ for $t>0$,

(iv) $\left(t \gamma^{\prime}(t)\right)^{\prime}$ is monotone increasing for $t>0$.

Let $\epsilon>0$, and define

$$
m(x, y)=\text { p.v. } \int_{-\infty}^{\infty} \exp i[x t+y \gamma(t)]\left(1+y^{2} t^{4} \gamma^{\prime \prime}(t)^{2}\right)^{1 / 4-\epsilon} \frac{d t}{t} .
$$

Then $m(x, y)$ is uniformly bounded on $L^{2}\left(\mathbf{R}^{2}\right)$.

PROOF. For each fixed $y$, let $t_{0}>0$ satisfy $|y| t_{0}^{2} \gamma^{\prime \prime}\left(t_{0}\right)=1$, and write

$$
m(x, y)=\text { p.v. } \int_{|t|<t_{0}}+\text { p.v. } \int_{|t|>t_{0}}=I+I I .
$$

Now $\left(1+y^{2} t^{4} \gamma^{\prime \prime}(t)^{2}\right)^{1 / 4-\epsilon}$ is monotone increasing in $t$ and is uniformly bounded for $|t| \leqslant t_{0}$. Hence by the second mean value theorem, and Theorem 2.2, the integral $\mathrm{I}$ is uniformly bounded in $x$ and $y$.

Next, set $H(t)=\int_{t_{0}}^{t} \exp i[x s+y \gamma(s)] d s$. Since $\gamma^{\prime \prime}$ is monotone decreasing, Van der Corput's lemma (Lemma 3.1) gives $|H(t)| \leqslant C\left(|y| \gamma^{\prime \prime}(t)\right)^{-1 / 2}$. Hence integrating by parts in II we obtain

$$
\begin{aligned}
|\mathrm{II}(x, y)| \leqslant & C \int_{|t|>t_{0}}\left(|y| \gamma^{\prime \prime}(t)\right)^{-1 / 2}\left(|y| t^{2} \gamma^{\prime \prime}(t)\right)^{1 / 2-2 \epsilon} \frac{d t}{t^{2}} \\
& +C \int_{|t|>t_{0}}\left(|y| \gamma^{\prime \prime}(t)\right)^{-1 / 2}\left[|y| t^{2} \gamma^{\prime \prime}(t)\right]^{-1 / 2-2 \epsilon} \frac{d}{d t}\left(y t^{2} \gamma^{\prime \prime}(t)\right) \frac{d t}{t} \\
= & R+S .
\end{aligned}
$$

Now

$$
\begin{aligned}
R & \leqslant C \int_{t_{0}}^{\infty} \frac{1}{\left[|y| t^{2} \gamma^{\prime \prime}(t)\right]^{2 \epsilon}} \frac{d t}{t} \\
& \leqslant \frac{C}{\left[|y| t_{0}^{\alpha} \gamma^{\prime \prime}\left(t_{0}\right)\right]^{2 \epsilon}} \int_{t_{0}}^{\infty} \frac{d t}{t^{1+(2-\alpha) 2 \epsilon}} \quad \text { by (ii) } \\
& \leqslant \frac{C}{\left[|y| t_{0}^{2} \gamma^{\prime \prime}\left(t_{0}\right)\right]^{2 \epsilon}}=C .
\end{aligned}
$$


Also

$$
S \leqslant C \int_{t_{0}}^{\infty} \frac{(d / d t)\left(|y| t^{2} \gamma^{\prime \prime}(t)\right)}{\left[|y| t^{2} \gamma^{\prime \prime}(t)\right]^{1+2 \epsilon}} d t \leqslant C \int_{1}^{\infty} \frac{d u}{u^{1+\epsilon}}=C .
$$

This completes the proof.

4. Examples. In this section, we construct the examples, mentioned in the introduction, of curves $\gamma(t)$ for which the truncated operators $T_{1}$ or $T_{3}$ fail to be bounded, even on $L^{2}\left(R^{2}\right)$.

THEOREM 4.1. There exists an odd, $C^{\infty}$-function $\gamma(t)$, defined for $-1 \leqslant$ $t<+1$, with $\gamma^{\prime \prime}(t) \geqslant 0$ for $t>0$ such that

$$
T_{1} f(x, y)=\text { p.v. } \int_{-1}^{+1} f(x-t, y-\gamma(t)) \frac{d t}{t}
$$

is not bounded on $L^{2}\left(R^{2}\right)$.

ProOF. Choose a sequence of positive numbers $\left\{a_{n}\right\}$ such that $a_{1} \leqslant 1$ and $a_{n+1} \leqslant a_{n} / 4(n+1)$. Let $\varphi(t)$ be an odd $C^{\infty}$-function on $[-1,+1]$ such that

(i) $\varphi(t)=0$ for $a_{n} \leqslant t \leqslant n a_{n}$ all $n \geqslant 1$,

(ii) $\varphi(t)>0$ for $(n+1) a_{n+1}<t<a_{n}$ all $n \geqslant 1$.

Put $\gamma(t)=\int_{0}^{t} \int_{0}^{s} \varphi(r) d r d s$ so that $\gamma^{\prime \prime}(t)=\varphi(t)$ on $[-1,+1]$. Note that $\gamma$ is odd and $C^{\infty}$ on $[-1,+1]$. Clearly $\gamma$, along with all its derivatives, vanishes at $t=0$, so we obtain

(a) $\gamma(t) \leqslant C t^{2}$ for $t>0$.

Also, since $\gamma^{\prime \prime}(t)=0$ on $\left[a_{n}, n a_{n}\right]$, there are real numbers $\lambda_{n}, \eta_{n}$ so that

(b) $\gamma(t)=\lambda_{n} t+\eta_{n}$ on $\left[a_{n}, n a_{n}\right]$.

To prove the theorem, it suffices to show that

$$
m_{1}(x, y)=\text { p.v. } \int_{-1}^{+1} \exp i[x t+y \gamma(t)] \frac{d t}{t}=(2 i) \int_{0}^{1} \sin (x t+y \gamma(t)) \frac{d t}{t}
$$

is unbounded on $\mathbf{R}^{2}$. To do this, we will show that there exists $C>0$ so that, for each $n$ there exists $\left(x_{n}, y_{n}\right)$ so that

$$
\int_{a_{n}}^{n a_{n}} \sin \left(x_{n} t+y_{n} \gamma(t)\right) \frac{d t}{t}=\log (n)
$$

while

$$
\left|\int_{t \in[0,1] ; t \notin\left[a_{n}, n a_{n}\right]} \sin \left(x_{n} t+y_{n} \gamma(t)\right) \frac{d t}{t}\right| \leqslant C .
$$

First note that if we let $x=-\lambda_{n} y$, then

$$
\int_{a_{n}}^{n a_{n}} \sin (x t+y \gamma(t)) \frac{d t}{t}=\int_{a_{n}}^{n a_{n}} \sin \left(y \eta_{n}\right) \frac{d t}{t}=\sin \left(y \eta_{n}\right) \log (n)
$$


and we can find arbitrarily large $y$ so that $\sin \left(y \eta_{n}\right)=+1$. Thus we are reduced to showing that for $y$ sufficiently large

$$
\left|\int_{0}^{a_{n}} \sin \left(-\lambda_{n} y t+y \gamma(t)\right) \frac{d t}{t}\right| \leqslant C
$$

and

$$
\left|\int_{n a_{n}}^{1} \sin \left(-\lambda_{n} y t+y \gamma(t)\right) \frac{d t}{t}\right| \leqslant C
$$

Note that

$\left|\int_{0}^{1 / \sqrt{ } y} \sin \left(-\lambda_{n} y t+y \gamma(t)\right) \frac{d t}{t}\right| \leqslant\left|\int_{0}^{1 / \sqrt{ } y} \sin \left(-\lambda_{n} y t\right) \frac{d t}{t}\right|+|y| \int_{0}^{1 / \sqrt{ } y} \gamma(t) \frac{d t}{t}$ $\leqslant C$ by (a).

Also note that $\left|\int_{1 / 2 a_{n}}^{a_{n}}\right|$ and $\left|\int_{n a_{n}}^{2 n a_{n}}\right|$ are each bounded by $\log 2$. Hence we are reduced to studying

$$
\int_{1 / \sqrt{ } y}^{1 / 2 a_{n}} \exp i\left[-\lambda_{n} y t+y \gamma(t)\right] \frac{d t}{t} \text { and } \int_{2 n a_{n}}^{1} \exp i\left[-\lambda_{n} y t+y \gamma(t)\right] \frac{d t}{t} \text {. }
$$

Now $-\lambda_{n} y+y \gamma^{\prime}(t)$ is monotone increasing and negative on the interval $[1 / \sqrt{ } y$, $\left.1 / 2 a_{n}\right]$. Hence if we put

$$
\varphi(s)=\int_{1 / \sqrt{ } y}^{s} \exp i\left[-\lambda_{n} y t+y \gamma^{\prime}(t)\right] d t,
$$

Van der Corput's lemma (Lemma 3.1) shows that $|\varphi(s)| \leqslant C /\left|y \gamma^{\prime}(s)-y \lambda_{n}\right|$. Thus, by the second mean value theorem we obtain

$$
\left|\int_{1 / \sqrt{ } y}^{1 / 2 a_{n}} \exp i\left[-\lambda_{n} y t+y \gamma(t)\right] \frac{d t}{t}\right|<\frac{C}{|y|^{1 / 2}\left(\left|\gamma^{\prime}\left(1 / 2 a_{n}\right)-\lambda_{n}\right|\right)} .
$$

Since $\gamma^{\prime \prime}$ is strictly positive on $\left((n+1) a_{n+1}, a_{n}\right),\left|\gamma^{\prime}\left(1 / 2 a_{n}\right)-\lambda_{n}\right|>0$, and hence this integral is uniformly bounded for all sufficiently large $y$. The term $\left|\int_{2 n a_{n}}^{1} \exp i\left[-\lambda_{n} y t+y \gamma(t)\right] d t / t\right|$ is handled similarly. This completes the proof.

THEOREM 4.2. Let $\psi(t)$ be any given positive increasing function of $t$. There exists an odd $C^{\infty}$-function $\gamma(t)$ defined for $|t| \geqslant 1$ such that $\gamma^{\prime \prime}(t) \geqslant \psi(t)$ for $t>0$ and such that

$$
T_{3} f(x, y)=\text { p.v. } \int_{|t|>1}^{\cdot} f(x-t, y-\gamma(t)) \frac{d t}{t}
$$

is unbounded on $L^{2}\left(R^{2}\right)$.

Proof. Pick a sequence of positive numbers $a_{n}$ so that $a_{1} \geqslant 1$ and $a_{n+1}$ 
$>4 n a_{n}$. For each $n$, choose $c_{n}$ so that $\psi(t) \leqslant c_{n}$ for $a_{n} \leqslant t \leqslant n a_{n}$. Let $\varphi(t)$ be an odd $C^{\infty}$-function defined for all $t$, which satisfies:

(a) $\varphi(t) \geqslant \psi(t)$ for $t \geqslant 1$.

(b) $\varphi(t)=c_{n}$ for $a_{n} \leqslant t \leqslant n a_{n}$.

(c) $\int_{1 / 2 a_{n}}^{a_{n}} \varphi(s) d s \geqslant c_{n} a_{n}+\left(n^{2}-1\right) c_{n} a_{n}^{2}, n=1,2, \ldots$.

(d) $\int_{a_{n}}^{2 n a_{n}} \varphi(s) d s \geqslant\left(n^{2}-1\right) c_{n} a_{n}^{2}$.

(e) $\int_{0}^{a_{n}} s \varphi(s) d s \geqslant \pi n^{2} c_{n} a_{n}^{2}$.

Let $\gamma(t)=\int_{0}^{t} \int_{0}^{s} \varphi(r) d r d s$, so that $\gamma^{\prime \prime}(t)=\varphi(t)$. To prove the theorem it suffices to show that

$$
m_{3}(x, y)=\int_{1}^{\infty} \sin (x t+y \gamma(t)) \frac{d t}{t}
$$

is unbounded on $\mathbf{R}^{2}$.

For each $n$, we can write

$$
\begin{aligned}
m_{3}(x, y) & =\int_{1}^{1 / 2 a_{n}}+\int_{1 / 2 a_{n}}^{a_{n}}+\int_{a_{n}}^{n a_{n}}+\int_{n a_{n}}^{2 n a_{n}}+\int_{2 n a_{n}}^{\infty} \\
& =\mathrm{I}+\mathrm{II}+\mathrm{III}+\mathrm{IV}+\mathrm{V} .
\end{aligned}
$$

Clearly $|\mathrm{II}|$ and $|\mathrm{IV}|$ are bounded by $\log (2)$. We will show that there exists $y_{n} \in\left(1 /\left(n^{2}-1\right) a_{n}^{2} c_{n}, 2 /\left(n^{2}-1\right) a_{n}^{2} c_{n}\right)$ so that if $x_{n}=-y_{n}\left[\int_{0}^{a_{n}} \varphi(s) d s-c_{n} a_{n}\right]$, then $\left|\mathrm{III}\left(x_{n}, y_{n}\right)\right| \geqslant C \log (n)$, while $\left|\mathrm{I}\left(x_{n}, y_{n}\right)\right| \leqslant C$ and $\left|\mathrm{V}\left(x_{n}, y_{n}\right)\right| \leqslant C$, where $C$ is independent of $n$.

For I and V, we consider $\int \exp i\left[x_{n} t+y_{n} \gamma(t)\right] d t / t$ where the integral is taken over the appropriate interval. The derivative of $f(t)=x_{n} t+y_{n} \gamma(t)$ is $x_{n}+y_{n} \gamma^{\prime}(t)$ which is negative and monotone increasing in $\left[1,1 / 2 a_{n}\right]$. Hence using the second mean value theorem, and Van der Corput's lemma (Lemma 3.1) we have

$$
\begin{aligned}
\left|\mathrm{I}\left(x_{n}, y_{n}\right)\right| & \leqslant \frac{C}{\left|x_{n}+y_{n} \gamma^{\prime}\left(1 / 2 a_{n}\right)\right|}=\frac{C}{y_{n}\left|c_{n} a_{n}-\int_{0}^{a_{n}} \varphi(s) d s+\int_{0}^{a_{n} / 2} \varphi(s) d s\right|} \\
& =\frac{C}{y_{n}\left|\int_{1 / 2 a_{n}}^{a_{n}} \varphi(s) d s-c_{n} a_{n}\right|} \leqslant \frac{C}{y_{n}\left(\left(n^{2}-1\right) a_{n}^{2} c_{n}\right)} \text { by (c) } \\
& \leqslant C
\end{aligned}
$$

since $y_{n} \in\left(1 /\left(n^{2}-1\right) a_{n}^{2} c_{n}, 2 /\left(n^{2}-1\right) a_{n}^{2} c_{n}\right)$.

Similarly, $\left|\mathrm{V}\left(x_{n}, y_{n}\right)\right|$ is bounded by 


$$
\begin{aligned}
\frac{C}{\left|x_{n}+y_{n} \gamma^{\prime}\left(2 n a_{n}\right)\right|} & =\frac{C}{y_{n}\left|\int_{0}^{2 n a_{n}} \varphi(s) d s-\int_{0}^{a_{n}} \varphi(s) d s+c_{n} a_{n}\right|} \\
& \leqslant \frac{C}{y_{n} \int_{a_{n}}^{2 n a_{n}} \varphi(s) d s} \leqslant \frac{C}{y_{n}\left(n^{2}-1\right) c_{n} a_{n}^{2}} \\
& \leqslant C
\end{aligned}
$$

if $y_{n} \geqslant 1 /\left(n^{2}-1\right) a_{n}^{2} c_{n}$.

Finally, for $t \in\left[a_{n}, n a_{n}\right]$, we have

$$
\gamma(t)=\frac{1}{2} c_{n} t^{2}+\left[\int_{0}^{a_{n}} \varphi(s) d s-c_{n} a_{n}\right] t+\left[\frac{1}{2} c_{n} a_{n}^{2}-\int_{0}^{a_{n}} s \varphi(s) d s\right] .
$$

Hence

$$
\int_{a_{n}}^{n a_{n}} \sin \left(x_{n} t+y_{n} \gamma(t)\right) \frac{d t}{t}=\int_{a_{n}}^{n a_{n}} \sin \left(\mu_{n}(t)\right) \frac{d t}{t}
$$

where

$$
\mu_{n}(t)=y_{n}\left[\frac{1}{2} c_{n} t^{2}+\left(\frac{1}{2} c_{n} a_{n}^{2}-\int_{0}^{a_{n}} s \varphi(s) d s\right)\right] .
$$

The change in $\mu_{n}(t)$ over $\left[a_{n}, n a_{n}\right]$ is $1 / 2 y_{n} c_{n}\left(n^{2}-1\right) a_{n}^{2} \leqslant 1$ for any $y_{n} \in$ $\left(1 /\left(n^{2}-1\right) a_{n}^{2} c_{n}, 2 /\left(n^{2}-1\right) a_{n}^{2} c_{n}\right)$. On the other hand,

$$
\mu\left(a_{n}\right)=y_{n}\left[c_{n} a_{n}^{2}-\int_{0}^{a_{n}} s \varphi(s) d s\right] \leqslant-y_{n} \pi\left(n^{2}-1\right) c_{n} a_{n}^{2} .
$$

Hence we can find $y_{n} \in\left(1 /\left(n^{2}-1\right) a_{n}^{2} c_{n}, 2 /\left(n^{2}-1\right) a_{n}^{2} c_{n}\right)$ so that $\left|\sin \left(\mu_{n}(t)\right)\right| \geqslant$ $\delta>0$ on the whole interval $a_{n} \leqslant t \leqslant n a_{n}$. This shows that $\left|\operatorname{III}\left(x_{n}, y_{n}\right)\right| \geqslant$ $\delta \log (n)$ and this completes the proof of the theorem.

Finally, we consider the operator $T_{3}$ associated to the curve $t \rightarrow(t, \log |t|)$.

For $t \geqslant 1$, we shall show, in fact, that the associated maximal function

$$
M f(x, y)=\sup _{h>1} \frac{1}{2} \int_{1}^{h} f(x,-t, y-\log t) d t
$$

is unbounded on $L^{p}\left(\mathbf{R}^{2}\right)$ for $p<\infty$. To see this, let $f(x, y)$ be the characteristic function of the rectangle $I_{N}=\{(x, y) \mid 0 \leqslant x \leqslant N, 0 \leqslant y \leqslant 1\}$. Then for every point $(x, y)$ such that $y \leqslant 0$ and $0 \leqslant x+e^{-y} \leqslant N$ and $0 \leqslant x+e^{1-y}<N$, we have $M f(x, y)>1-e^{-1}$. If $M$ were bounded on $L^{p}\left(\mathbf{R}^{2}\right)$ we would have

$$
m\left\{(x, y) \mid M f(x, y)>1-e^{-1}\right\} \leqslant C\|f\|_{p}^{p} \leqslant C N .
$$

But an easy calculation shows that the measure of the set of points when $y<$ $0,0 \leqslant x+e^{-y} \leqslant N$ and $0 \leqslant x+e^{1-y} \leqslant N$ is bigger than $C N \log (N)$. Hence $M$ is unbounded on $L^{p}\left(\mathbf{R}^{2}\right)$. One can show that the operator 


$$
T_{3} f(x, y)=\text { p.v. } \int_{|t| \geqslant 1} f(x-t, y-\log |t|) \frac{d t}{t}
$$

is unbounded on $L^{p}\left(R^{2}\right)$ in a similar way.

\section{REFERENCES}

[NRW] A. Nagel, N. Rivière and S. Wainger, Singular integrals along curves. II, Amer. J. Math. (to appear).

[S] E. M. Stein, Singular integrals and differentiability properties of functions, Princeton Univ. Press, Princeton, N.J., 1970. MR 44 \#280.

[SW] E. M. Stein and G. Weiss, Introduction to Fourler analysis on Euclidean spaces, Princeton Univ. Press, Princeton, N.J., 1971. MR 46 \#4102.

[Z] A. Zygmund, Trigonometric series, Vol. I, 2nd rev. ed., Cambridge Univ. Press, New York, 1959. MR 21 \#6498.

DEPARTMENT OF MATHEMATICS, UNIVERSITY OF WISCONSIN, MADISON, WISCONSIN 53706 